Trauma Berufskrankh 2005 · 7[Suppl 1]:S105-S109 DOI 10.1007/s10039-004-0908-3

Online publiziert: 7. Juli 2004

(c) Springer-Verlag 2004

\author{
M. Bühler ${ }^{1}$ S. Kirschner ${ }^{2}$ \\ ${ }^{1}$ Abteilung für Septische Chirurgie, BG-Unfallklinik, Frankfurt am Main \\ ${ }^{2}$ Orthopädische Universitätsklinik König-Ludwig-Haus, Julius-Maximilian-Universität, \\ Würzburg
}

\title{
Therapiekonzepte bei Osteitis
}

Organsystem „Knochen“. Der Knochen ist ein hoch spezialisiertes Organsystem. Durch die simultane Aktivität von Osteoblasten und Osteoklasten unterliegt er einer ständigen Anpassung an die mechanischen Anforderungen. Die notwendige Stabilität wird durch Mineralisation der Grundsubstanz erreicht. Dieser Vorgang benötigt mehr Zeit als vergleichbare Reparaturvorgänge an anderen Gewebearten. Der Knochen ist als bradytrophes Gewebe einzustufen und bedarf im Fall einer Infektion einer besonderen Behandlung.

\section{Keimspektrum}

Staphylococcus aureus und Staphylococcus epidermidis stellen die häufigsten Erreger septischer Komplikationen am Bewegungsapparat dar. In der Erregerstatistik der Abteilung für Septische Chirurgie der BG-Unfallklinik Frankfurt am Main machten sie $68 \%$ der infektauslösenden Keime aus. Der Anteil von MRSA ist mit 5\% vergleichsweise gering.

Staphylokokken sind durch ihre große Anpassung an den Menschen und ihre besonderen Eigenschaften als Infekterreger am Bewegungsapparat besonders erfolgreich. Sie haften an Kunststoff- und Metalloberflächen durch Ausbildung eines Biofilms. Dieser besteht aus Bakterien und extrazellulären Polysacchariden. In ihm sind die Staphylokokken für eingesetzte Antibiotika schlecht oder gar nicht erreichbar (• Abb. 1).

Darüber hinaus können die Keime ihren Stoffwechsel regulieren, ein Teil der
Population geht in eine planktonische Dauerform über, die einer pharmakologischen Therapie weitgehend unzugänglich ist.

Bei rezidivierenden Infektionen von Osteitiden hat man eine besondere Staphylokokkenvariante, die so genannten Small colony variants gefunden. Diese SCV sind durch reduziertes Wachstum, eingeschränkte Exotoxinproduktion und die Möglichkeit der intrazellulären Persistenz gekennzeichnet. Eine erfolgreiche Anzucht dieser Keime setzt ein spezielles Medium und verlängerte Bebrütungszeiten voraus. Zunächst hat eine solche Infektion weniger stark ausgeprägte klinische Symptome als eine purulente Staphylokokkeninfektion. Sie erzeugt jedoch eine dauerhafte Inflammation, die zu rezidivierenden Infekten oder vorzeitiger Implantatauslockerung führen kann.

Daneben stellen Pseudomonaden und Streptokokken weitere wichtige Erreger dar. Pseudomonas aeruginosa ist ebenfalls in der Lage, einen Biofilm zum Selbstschutz vor Antibiotika und der zellulären Abwehr zu bilden.

\section{Erkennen, Bekennen, Handeln}

Der Schlüssel der erfolgreichen Osteitisbehandlung liegt zunächst einmal darin, diese Komplikation als solche überhaupt zu erkennen. Osteitiden, die im postoperativen Verlauf auftreten, müssen gegen den normalen postoperativen Verlauf abgegrenzt werden (• Abb. 2). Fulminante Infektionen mit Fieber, putridem Wundsekret und starken Schmerzen stellen in tionen, tritt meist bei Kindern auf
bei Erwachsenen die Ausnahme. 


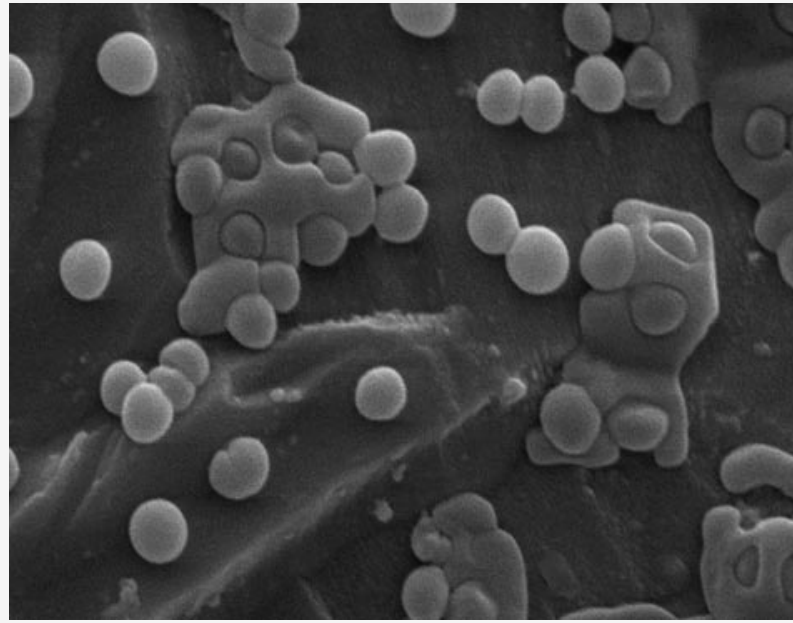

Abb. 1 Elektronenmikroskopische Aufnahme (Vergr. 10.000:1) von Biofilm bildenden Staphylokokken auf einer Titanoberfläche

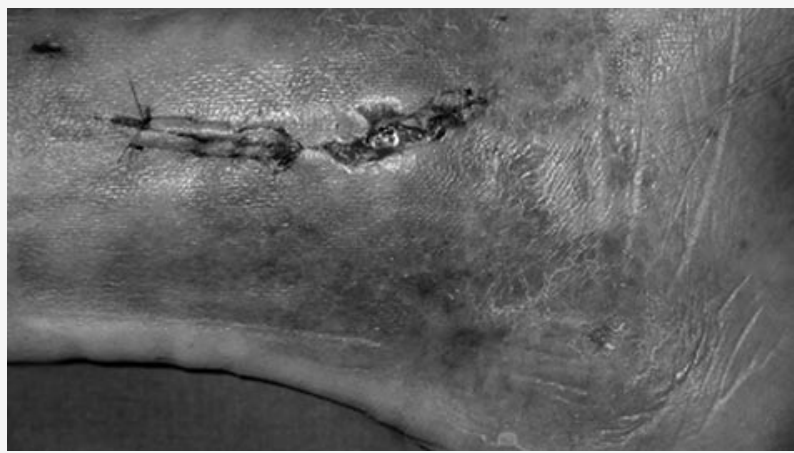

Abb. $3 \Delta$ Klinischer Aspekt medialer Unterschenkel, Wundheilungsstörung Malleolus medialis mit putrider Wundsekrektion und frei liegendem Osteosynthesematerial
Abb. $2>$ Postoperative Kontrolle nach Versorgung einer kompletten Unterschenkelfraktur mit Beteiligung des Pilons

Abb.4 Postopera-
tive Kontrolle nach
Durchführung der ers-
ten Etappenrevision,
vollständige Entfer-
nung der ausgelocker-
ten Osteosynthese,
Débridement und Ne-
krosektomie der dista-
len Tibia, Einlage von
Septopalketten

Septopalketten
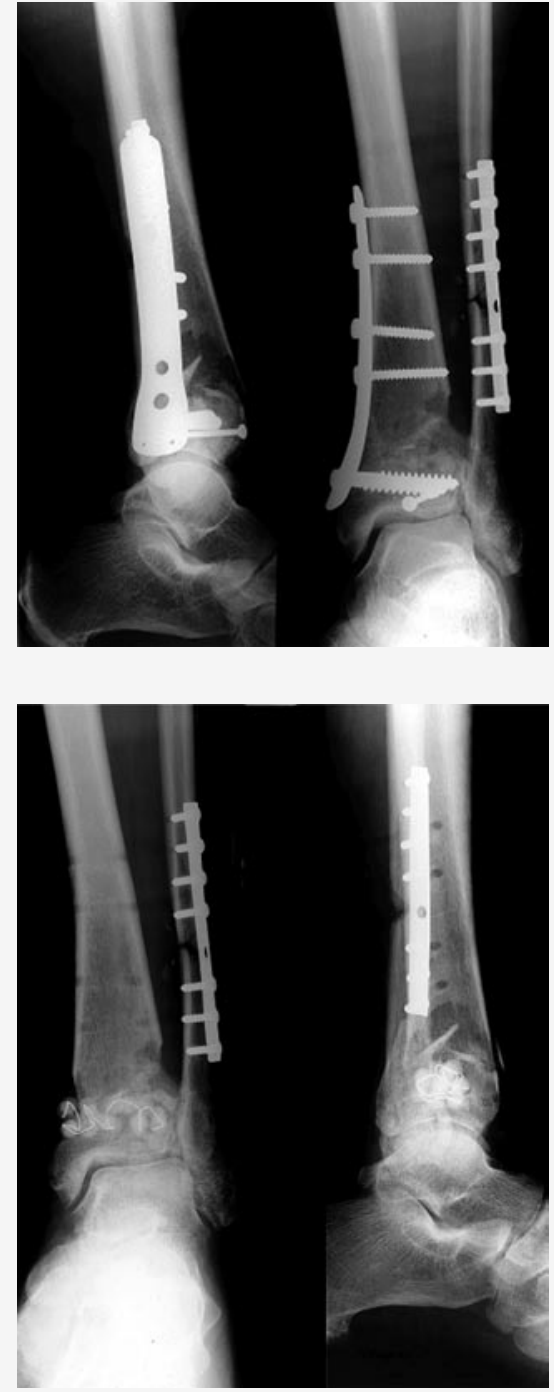

der Regel keine diagnostischen Schwierigkeiten dar. Bei Patienten, die noch nicht alle Zeichen einer septischen Komplikation zeigen, ist die richtige Diagnosestellung schwierig. Häufig ist der Operateur geneigt, die Symptomatik nicht als Komplikation zu bewerten, da das Eintreten einer septischen Komplikation häufig als persönliches Versagen betrachtet wird und eine entsprechende psychologische Barriere das Erkennen erschwert. Eine Möglichkeit einer adäquaten Diagnosestellung ist die Vorstellung des Patienten bei einem erfahrenen Kollegen, der nicht in die Behandlung mit eingebunden ist (• Abb. 3).

Dem Erkennen folgt das Bekennen zur Komplikation, und erst jetzt wird die weitere Osteitistherapie möglich. Ein frühzeitiger Behandlungsbeginn sollte angestrebt werden, auch wenn im Rahmen der ersten operativen Revision die Abgrenzung von avitalem Gewebe häufig noch schwierig ist. Der Übergang von einer akuten in eine chronische Osteitis sollte durch raschen Therapiebeginn vermieden werden [4].

Die Behandlung der Osteitis erfolgt in erster Linie operativ. Sie sollte durch einen erfahrenen Operateur vorgenommen werden. Die Durchführung sämtlicher operativer Schritte, wie z. B. der Verfahrenswechsel der Osteosynthese oder eine möglicherweise erforderliche intensivmedizinische Betreuung, sollten vor Ort möglich sein $[1,7,16]$.

Die erste Revisionsoperation dient neben der Infektberuhigung und der Gewinnung von Material zur mikrobiologischen Untersuchung auch der vollständigen Bestandaufnahme: Das Implantat wird auf Festigkeit überprüft, das Ausmaß des Infekts im Rahmen der Sequesterektomie und des Weichteildébridements präzise eingeschätzt. Auf Grundlage dieser Informationen muss die weitere Therapie festgelegt werden. Die realistisch erreichbaren Behandlungsergebnisse müssen transparent gemacht und der damit verbundene Aufwand dem Patienten dargestellt werden.

Hofmann et al. [8] haben die psychischen und sozialen Auswirkungen der Extremitätenrekonstruktion mit einer Amputation verglichen. In dieser Studie konnte nicht bei allen Patienten der geplante Extremitätenaufbau erreicht werden. Die Behandlungszeiten und deren Auswirkungen auf soziale Lebensumstände waren in der Gruppe der Patienten mit Amputationen deutlich geringer. Langfristig zeigte sich ein Vorteil bei Mobilisierung und Berufsfähigkeit für die Patientengruppe mit Extremitätenaufbau. Die Unterschenkelamputation stellt einen weit reichenden 
Eingriff dar, der nicht jedem Patienten mit Hinweis auf die verkürzten Behandlungszeiten empfohlen werden kann.

\section{Therapieplanung}

Die Osteitis muss nach Diagnosestellung in ihrer Bedeutung eingeordnet werden. Die Unterteilung in

- ein Problem,

- eine leichte und

- schwere Komplikation

hat sich im klinischen Alltag bewährt. Osteitiden stellen leichte oder schwere Komplikationen dar [4].

Osteitis als leichte Komplikation. Eine Beruhigung ist mit konservativen Maßnahmen nicht zu erreichen. In günstigen Fällen einer leichten Komplikation kann versucht werden, die Situation mit einem operativen Eingriff zu beruhigen. Als Beispiel kann der postoperative Infekt nach Plattenosteosynthese genannt werden. In diesem Fall kann ein Débridement mit lokaler und systemischer Antibiotikatherapie als einzeitige Maßnahme erfolgreich sein [9, 10, 11, 12]. Nach Frakturkonsolidierung kann die Septopalkette im Rahmen der Materialentfernung entnommen werden.

Osteitis als schwere Komplikation. Bei schweren Komplikationen ist stets ein unverzügliches, konsequentes und mehrzeitiges operatives Vorgehen erforderlich. Der erste Schritt der Behandlung schwerer Infektionen stellt die Beruhigung der Infektion dar. Im Rahmen des erforderlichen radikalen Débridements müssen eine vollständige Nekrosektomie und Entfernung von Knochensequestern durchgeführt werden (• Abb. 4). Hierbei werden Gewebeproben zur mikrobiologischen Untersuchung und zur histopathologischen Begutachtung gewonnen. Bei schweren Osteitiden kommt es regelhaft zur Entstehung von Knochen-, aber auch Weichteildefekten. Bei schweren postoperativen Osteitiden wird man meist eine instabile Osteosynthese vorfinden, die einen Verfahrenswechsel auf einen Fixateur externe erfordert (• Abb. 5) [16].

Nach Abschluss des Débridements erfolgt eine Säuberung der Wunde mit
Trauma Berufskrankh 2005 · 7[Suppl 1]:S105-S109

DOI 10.1007/s10039-004-0908-3

(c) Springer-Verlag 2004

\section{Bühler · S. Kirschner}

\section{Therapiekonzepte bei Osteitis}

\section{Zusammenfassung}

Trotz ständig verbesserter medizinischer Versorgung kommt es weiterhin zu Osteitiden, posttraumatischen Knocheninfektionen. Sie entwickeln sich als postoperative Komplikation oder auch nach offenen Extremitätenverletzungen und bedürfen eines befundadaptierten Behandlungskonzepts. Wesentliche Infektionserreger sind Staphylokokken. Die Osteitis ist nur bei frühzeitiger adäquater Therapie zu beherrschen. Nach der aufgrund verschiedener psychologischer Mechanismen erschwerten Diagnosestellung und der ersten operativen Revision kann die weitere - chirurgisch erfolgende - Therapie geplant werden. Befundadaptiert umfasst sie ein großes Spektrum vom einfachen Débridement bis zum Verfahrenswechsel und Weichteil-

\section{Treatment schedules in osteitis}

\section{Abstract}

Despite constant improvements in medical care, osteitis - posttraumatic infection of bone - is still encountered. It develops as a postoperative complication or after open injuries of the extremities and requires a treatment schedule that is tailored to the findings. The main pathogens are staphylococci. Osteitis cannot be controlled unless an adequate therapy is implemented early in its course. Once the initial diagnosis has been made - various psychological mechanisms make this difficult - and the first surgical revision carried out the further surgical therapy can be planned. This will depend on the findings, and the options range from simple debridement through switching to a different treatment method to soft tissue trans- transfer. Die Behandlung sollte durch erfahrene Operateure an Zentren erfolgen. Ein günstiges Behandlungsergebnis für den Verletzten kann nur durch enge Verzahnung der operativen Eingriffe mit der rehabilitativen Nachbehandlung erreicht werden. Bei bleibenden Funktionseinschränkungen müssen Hilfsmittel zeitnah angepasst werden. Die Behandlung der Osteitis übersteigt die Kosten unkomplizierter Frakturen bei weitem.

\section{Schlüsselwörter}

Osteitis .

Befundadaptiertes Behandlungskonzept . Interdisziplinäre Zusammenarbeit . Rehabilitative Nachbehandlung · Hilfsmittel

plantation. The treatment should be carried out by experienced surgeons in specialist centres. A good outcome of treatment for the injured person is only possible if the surgical interventions are closely dovetailed with subsequent rehabilitation. If function remains restricted, specially adapted aids must be prescribed, which must be checked frequently to see whether further adaptations are needed. The treatment of osteitis is far more costly than that of uncomplicated fractures.

\section{Keywords}

Tailored treatment schedule . Interdisciplinary cooperation . Aftertreatment and rehabilitation 


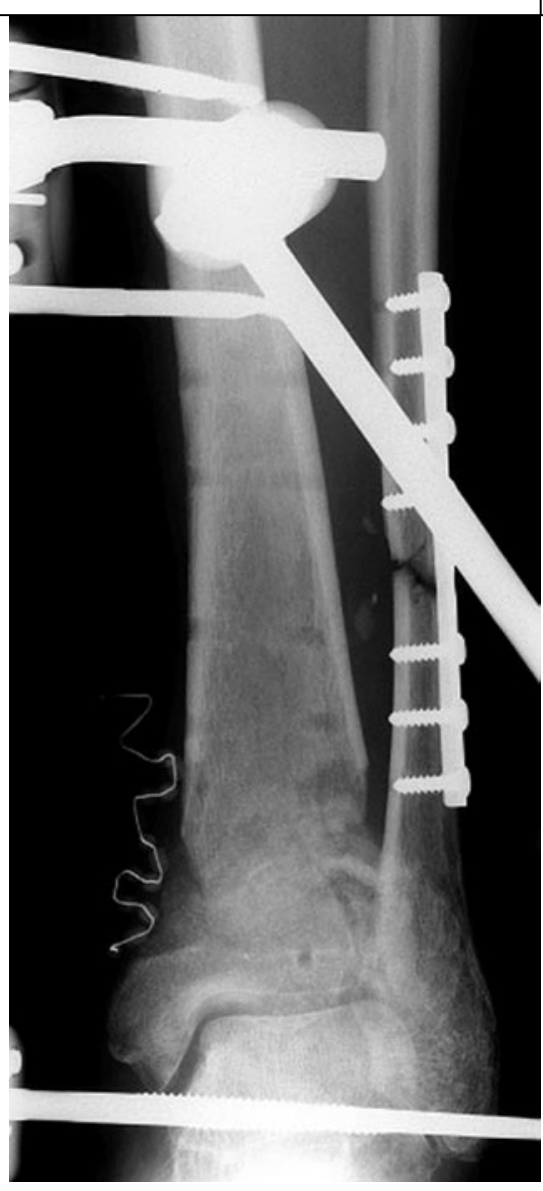

Abb. $5 \Delta$ Sekundäre Stabilisierung mit Fixateur externe, Spongiosaplastik, lokale Antibiotikatherapie

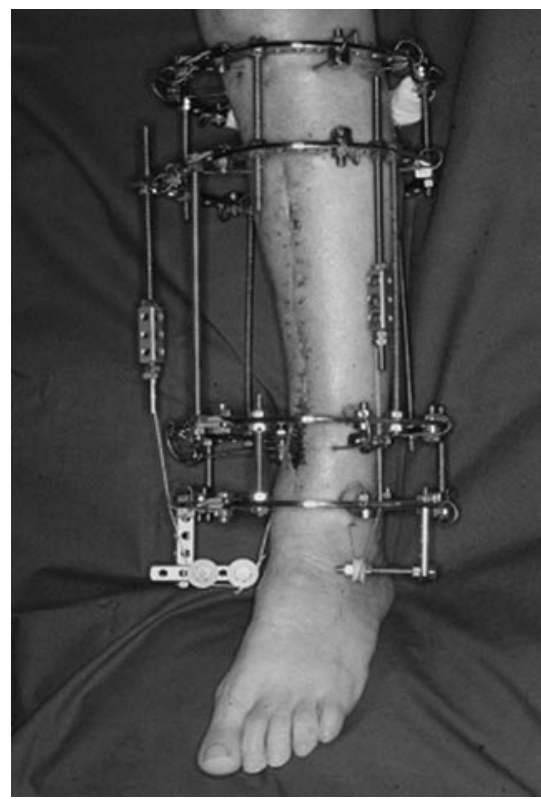

Abb. $6 \Delta$ Klinisches Beispiel Kallusdistraktion: Anlage eines Ilisarov-Ringfixateurs und Vorbereitung zur Kallusdistraktion mit Kabelrollen nach Prof. Weber (Aachen)

Infektmanagement

Jetlavage (pulsierende Druckspülung, mindestens 51 Ringer-Laktat-Lösung) [15]. Die entstandenen Hohlräume werden mit lokalen Antibiotikaträgern wie z. B. Septopalketten aufgefüllt $[3,10]$. Die Wunde wird nach Einlage von Überlaufdränagen verschlossen. Sofern die vorhandenen Weichteile den Wundverschluss nicht gestatten, wird der vorübergehende Hautverschluss mit Kunsthaut durchgeführt oder eine Dermatotraktion [2] und Wundgrundkonditionierung mittels Vakuumversiegelung erzeugt [5]. Postoperativ erfolgt eine kalkulierte systemische Antibiotikatherapie, nach Erhalt der mikrobiologischen Ergebnisse mit Antibiogramm muss die kalkulierte Antibiotikatherapie ggf. modifiziert werden.

Der weitere Behandlungsverlauf bei schweren Osteitiden ist stark von der Situation des individuellen Patienten geprägt. Eine Beschreibung des weiteren Behandlungsablaufs kann daher nicht in allgemeiner Form erfolgen. Große Bedeutung kommt einer kontinuierlichen Patientenbetreuung zu, da unterschiedliche Untersucher häufig abweichende Einschätzungen entwickeln. Hieraus würden sich wechselnde Behandlungen und unterschiedliche Behandlungsprioritäten ergeben. Bei Patienten mit schweren Osteitiden sollten solche Situationen vermieden werden.

Nach erfolgreicher Infektberuhigung müssen die verbliebenen Probleme erfasst und in ihrer Bedeutung und Behandlungsindikation festgelegt werden. Ein Knochenaufbau, z. B. per Kallusdistraktion (• Abb. 6), kann nur erfolgreich sein, wenn u. a. eine leistungsfähige Durchblutungssituation vorhanden ist [1].

Sofern Weichteildefekte vorliegen, muss zunächst die Durchführung einer Lappenplastik zur Verbesserung der lokalen Situation in Betracht gezogen werden [4]. Die Planung erfolgt also in Behandlungsabschnitten. Der Patient sollte intensiv in die Planung einbezogen werden. Seine Mitarbeit ist für den Behandlungserfolg von wesentlicher Bedeutung. Gemeinsam gilt es, individuelle Lösungen zu finden, die z. B. auch eine Amputation umfassen können, obwohl ein vollständiger Extremitätenaufbau medizinisch möglich wäre.

\section{Rehabilitation}

Ihr kommt bei Patienten mit Osteitiden große Bedeutung zu. Sie beginnt mit krankengymnastischer Übungsbehandlung bereits am 2. postoperativen Tag und schließt sich an jede Revision an. Nur durch eine kontinuierliche physiotherapeutische Betreuung der Patienten kann der Entstehung großer funktioneller Defizite entgegengewirkt werden. Im direkten postoperativen Verlauf kann es erforderlich werden, Krankengymnastik, Narkosemobilisation und invasive Schmerztherapieverfahren zu kombinieren.

Die Versorgung der Patienten umfasst jedoch mehr als die reine krankengymnastische Übungsbehandlung: Die Auswahl geeigneter Hilfsmittel und deren zeitnahe Anpassung schaffen die Voraussetzung zu einer schnellen Rehabilitation. Durch die ergotherapeutische Betreuung erlernt der Patient Techniken, um funktionelle Einschränkungen im täglichen Leben zu überwinden und die angepassten Hilfsmittel sinnvoll einzusetzen. Physikalische Therapiemaßnahmen erweitern die Behandlungsmöglichkeiten. Im Fall lebensverändernder Behandlungsfolgen, wie z. B. einer Unterschenkelamputation, ist eine professionelle psychologische Mitbetreuung der Patienten erforderlich.

Nur bei wenigen Patienten, die wegen einer Osteitis behandelt wurden, kann die Arbeitsfähigkeit kurzfristig wieder erreicht werden. Hier besteht die Möglichkeit, die Eingliederung stufenweise (Belastungserprobung) unter Aussparung bestimmter belastender Tätigkeiten vorzunehmen. Alternativ kann im Rahmen des BG-lichen Heilverfahrens eine stationäre Weiterbehandlung als BGSW erfolgen.

Die aufgezeigten Maßnahmen einer umfassenden Rehabilitation können unter dem gegenwärtigen Kostendruck, der durch die Einführung der DRG entstanden ist, nur im BG-lichen Heilverfahren angeboten werden.

\section{Kosten}

Die Behandlung von Osteitiden stellt eine große Herausforderung dar. Sie ist v. a. durch verlängerte Behandlungsdauer und großen finanziellen Aufwand gekennzeichnet. Die mit der Erkrankung verbundenen 
Tabelle 1

\section{Kosten Unterschenkelfraktur unkompliziert vs. Unterschenkelosteitis}

\begin{tabular}{|lcc} 
& $\begin{array}{l}\text { Unkomplizierte } \\
\text { Unterschenkelfraktur }\end{array}$ & $\begin{array}{l}\text { Unterschenkel- } \\
\text { osteitis }\end{array}$ \\
\hline Behandlungskosten [€] & 7500 & 75.000 \\
\hline Dauer der stationären Behandlung [Tage] & 14 & 156 \\
\hline Dauer der Arbeitsunfähigkeit [Wochen] & 8 & 104 \\
\hline
\end{tabular}

Arbeitsunfähigkeitszeiten übersteigen die einer unkomplizierten Unterschenkelfraktur bei weitem. Da trotz des enormen Ressourceneinsatzes in der Regel kein ideales Ergebnis erreicht werden kann, muss das Hauptaugenmerk in der täglichen Praxis auf der Komplikationsprävention liegen (- Tabelle 1).

\section{Fazit für die Praxis}

Die posttraumatische Osteitis tritt trotz verbesserter Behandlungsbedingungen am Bewegungsapparat weiterhin auf. Sie stellt eine schwer wiegende Komplikation mit weit reichenden Folgen dar. Die Therapie der Osteitis wird erst nach Erkennen dieser Komplikation möglich. Der Erkennungsprozess wird durch verschiedene psychologische Barrieren erschwert und damit ein frühzeitiger Behandlungsbeginn häufig gefährdet. Die Behandlung der Osteitis ist zeit- und kostenintensiv. Sie kann nur mit einem konsequenten Behandlungskonzept unter Einbeziehung des Patienten erfolgreich sein. Das Behandlungsspektrum umfasst

\section{- Etappenrevisionen mit Débridement und Sequesterektomie, \\ - Verfahrenswechsel der Osteosynthe- se, \\ - temporären Wundverschluss durch Va- kuumversieglung oder Kunsthautde- ckung, \\ - freien Gewebetransfer und Kallusdis- traktion, aber auch \\ - die Amputation einer Gliedmaße.}

Die Behandlung von Osteitiden setzt große Erfahrung des Therapeuten und seines Teams voraus und sollte in Zentren mit entsprechenden Behandlungsmöglichkeiten durchgeführt werden.

\section{Korrespondierender Autor \\ Dr. M. Bühler}

Abteilung für Septische Chirurgie, BG-Unfallklinik, Friedberger Landstraße 430, 60389 Frankfurt am Main E-Mail:buehlersc@hotmail.com

Interessenkonflikt: Der korrespondierende Autor versichert, dass keine Verbindungen mit einer Firma, deren Produkt in dem Artikel genannt ist, oder einer Firma, die ein Konkurrenzprodukt vertreibt, bestehen.

\section{Literatur}

1. Aronson J, Johnson E, Harp JH (1989) Local bone transportation for treatment of intercalary defects by the llizarov technique - biomechanical and clinical considerations. Clin Orthop 243: 71-79

2. Bohm HJ, Hierholzer G, Strich R (1994) [Dynamic skin suture for closure of the incision defect after compart-

3. Buchholz HW, Engelbrecht H (1970) Über die Depotwirkung einiger Antibiotika bei Vermischung mit dem Kunstharz Palacos. Chirurg 41: 511-515

4. Bühler M, Engelhardt M, Schmidt HGK (2003) Septische postoperative Komplikationen. Atlas für Unfallchirurgen und Orthopäden. Springer, Berlin Heidelberg New York

5. Fleischmann W, Russ M, Marquardt C (1996) [Closure of defect wounds by combined vacuum sealing with instrumental skin expansion]. Unfallchir-

6. Heppert V, Wentzensen A (1998) Treatment of soft tissue damage - definitive management. Langenbecks Arch Chir Suppl 115: 964-967

7. Hofmann GO, Bär T, Bühren V (1997) Osteosyntheseimplantat und früher postoperativer Infekt: Sanierung mit oder ohne Materialentfernung. Chirurg 68: 1175

8. Hofmann GO, Nikutta M, Pöppel E (2002) GliedmaBenerhalt oder Amputation bei chronischer Osteitis am Unterschenkel: psychosoziale Folgen eines protrahierten Behandlungsverlaufes. Aktuelle Traumatol 32: 111-123

9. Kirschner S, Bühler M (2004) Der Stellenwert von Septocoll in der Behandlung von Knochen- und Weichteilinfektionen. Eine Anwendungsbeobachtung. In: Langendorff HU (Hrsg) Homo reparandus. Dortmunder Unfallchirurgie und Orthopädie-Tagung, Dortmund

10. Klemm K (1976) Die Behandlung chronischer Knocheninfektion mit Gentamicin-PMMA-Kugelketten und -kugeln. Unfallchirurg 1:20-25

11. Klemm K (2001) The use of antibiotic-containing bead chains in the treatment of chronic bone infec tions. Clin Microbiol Infect 7: 28-31 ment incision]. Aktuelle Traumatol 24: 140-144 urg 99: 970-974
12. Klemm K, Börner M (1986) Behandlung der chronischen Osteomyelitis mit Gentamicin-PMMA-Ketten. Unfallchirurg 12: 128-131

13. Mader JT, Mohan D, Calhoun J (1997) A practical guide to the diagnosis and management of bone and joint infections. Drugs 54: 253-264

14. Patzakis MJ, Mazur K, Wilkins J et al. (1993) Septopal beads and autogenous bone grafting for bone defects in patients with chronic osteomyelitis. Clin Orthop 295: 112-118

15. Saxe A, Goldstein E, Dixon S et al. (1980) Pulsatile lavage in the management of postoperative wound infections. Am Surg 46: 391-397

16. Schmidt HGK (1991) Fixateur externe und Infekt. In: Wolter D, Zimmer W (Hrsg) Die Plattenosteosynthese und ihre Konkurenzverfahren. Springer, Berlin Heidelberg New York, S 286-295

17. Ziebuhr W, Krimmer V, Hoffmann A et al. (2001) Molecular diagnosis of septicemic loosening of orthopaedic implants. Nova Acta Leopoldina 321: 91101 